\title{
Erratum: Luminosity distance and anisotropic sky-sampling at low redshifts: A numerical relativity study [Phys. Rev. D 104, 023525 (2021)]
}

\author{
Hayley J. Macpherson® and Asta Heinesen
}

(Received 24 September 2021; published 12 November 2021)

\begin{abstract}
In this erratum we fix a bug in the generation of initial conditions for the simulations presented in our paper "Luminosity distance and anisotropic sky sampling at low redshifts: A numerical relativity study." Our new simulations have overall smaller typical density contrasts, and therefore the amplitude of anisotropic effects found has reduced. However, qualitatively our results are unchanged.
\end{abstract}

DOI: 10.1103/PhysRevD.104.109901

\section{INTRODUCTION}

This is an erratum to our paper "Luminosity distance and anisotropic sky-sampling at low redshifts: A numerical relativity study," published in Phys. Rev. D [[1] hereafter MH21]. In MH21 we investigated the role of low-redshift anisotropy in cosmological simulations using numerical relativity. Unfortunately, there was a bug in the code used to generate the initial data for these simulations. This bug caused a significant change to the power spectrum of density fluctuations at redshift $z=0$.

In MH21 we used the CLASS ${ }^{1}$ matter power spectrum at $z=1000$ to generate the initial density fluctuations, $\delta$, from which we calculated the initial metric perturbation $\phi$ and velocity field $v^{i}$ of the matter in the longitudinal gauge (see [2] for more details of this process). The bug was present in a constant used to calculate both $\phi$ and $v^{i}$, resulting in incorrect initial power spectra for these perturbations. This resulted in an overall increase in amplitude of the initial $v^{i}$ power spectrum, and a slight change in slope of the initial $\phi$ power spectrum. These errors propagated and ultimately resulted in an incorrect spectral shape for the density power spectrum at large scales at $z=0$. Correcting this bug reduces the typical density contrasts on the scales we studied in MH21.

We have therefore rerun both simulations used in $\mathrm{MH} 21$ with the bug fixed, and in this erratum we recreate all figures and tables from MH21 using these updated simulations.

\section{UPDATED RESULTS}

Here we present the same results as in MH21 using the updated simulation with $200 \mathrm{~h}^{-1} \mathrm{Mpc}$ coarse-graining scale. In addition, we include the same figures reproduced for the simulation with $100 \mathrm{~h}^{-1} \mathrm{Mpc}$ coarse-graining scale in the Appendix. Because of the reduced density contrasts these results are more similar to the main results presented in $\mathrm{MH} 21$ for the $200 h^{-1}$ Mpc smoothing case.

\section{A. Updated simulation snapshots}

Figure 1 shows two-dimensional slices of the rest-mass density, expansion rate, shear, and acceleration (top-left to bottom-right panels, respectively) in the updated simulation with box size $L=25.6 h^{-1} \mathrm{Gpc}$ and resolution $N=128$, i.e., with $200 \mathrm{~h}^{-1} \mathrm{Mpc}$ coarse-graining scale. This is to be compared with Fig. 1 in MH21. We now see significantly less largescale power present in all fluid variables, and our simulations now better reflect the statistical homogeneity and isotropy we expect on large scales. Typical density contrasts in our updated simulations are smaller than those in MH21, by a factor of $\sim 3$ on $200 h^{-1} \mathrm{Mpc}$ scales. As we discussed in Sec. IV of MH21, this reduction in density contrasts of the model universe will reduce the amplitude of anisotropic effects measured by our observers. However, we will show that, as expected, our results qualitatively remain unchanged.

\section{B. Sky variance of observational effective parameters}

Here we reassess the level of anisotropy measured across individual observer's skies in the updated simulations. Figure 2 shows an example sky map of the observational effective parameters for one observer in the updated simulation with

\footnotetext{
${ }^{1}$ http://class-code.net.
} 

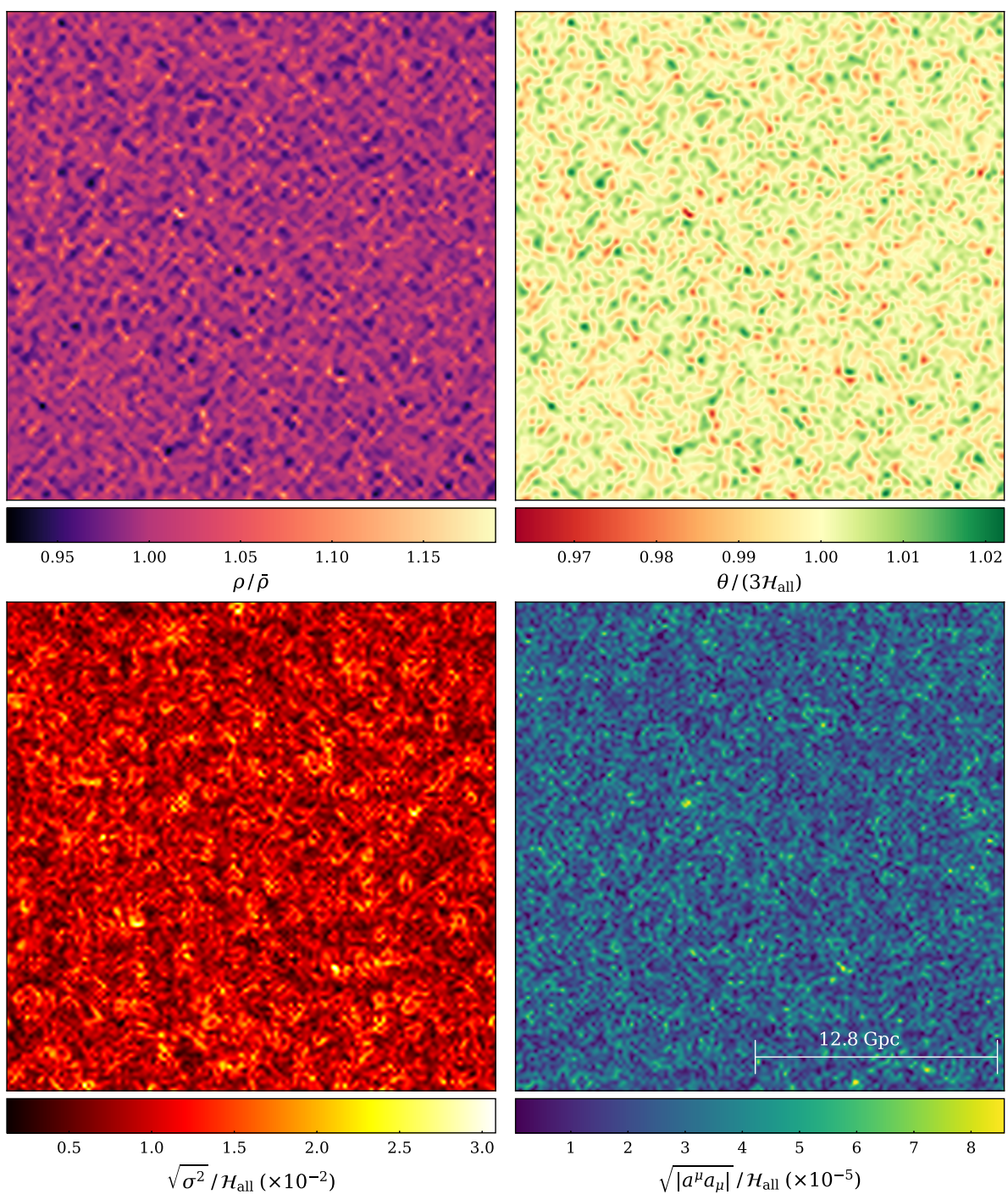

FIG. 1. Rest-mass density, expansion rate, shear, and acceleration (top-left to bottom-right panels, respectively) at $z \approx 0$. This is for comparison with Fig. 1 of MH21. We show two-dimensional slices through our updated simulation with $25.6 h^{-1} \mathrm{Gpc}$ box size and $200 h^{-1}$ Mpc coarse-graining scale.

$200 \mathrm{~h}^{-1}$ Mpc smoothing scale. ${ }^{2}$ This is for rough comparison with Fig. 3 in MH21, though we note that it is not the same observer. The amplitudes of anisotropic signatures for our observers have reduced relative to the amplitudes found in MH21. However, qualitatively we see that the same multipoles are dominating the anisotropic signals of each parameter. That is, with the quadrupole dominating the anisotropic signal of $\mathfrak{H}_{o}$, the dipole dominating $\mathfrak{Q}_{o}$ and $\mathfrak{R}_{o}$, and the quadrupole dominating $\mathfrak{J}_{o}$. The anisotropic variance in the effective Hubble parameter has reduced to $1.5-2 \%$, which may be expected based on the reduced density contrasts. We still see $\sim 85 \%$ variance in the effective deceleration parameter, and $\sim 40 \%$ variance in the effective curvature parameter across this observers sky.

Figure 3 shows the maximal sky variance, $\Delta$, for 1000 observers with a "FullSky" sampling with 300 lines of sight (see Fig. 2 in MH21 for a representation of the "FullSky" distribution). This is for direct comparison with Fig. 4 in MH21. The level of anisotropy still does not depend on the local density $\delta_{o}$ at the observer position within a particular model universe. However, typical sky variances for observers have reduced relative to MH21. Our conclusion that an observer's measurement of local anisotropy is independent of the density contrast at their position in the Universe is therefore robust.

In Table I we quote the updated average and maximum values of $\Delta$ over all 1000 observers in simulations with both $100 \mathrm{~h}^{-1} \mathrm{Mpc}$ and $200 \mathrm{~h}^{-1} \mathrm{Mpc}$ coarse-graining scales. This is for comparison with Table I in MH21.

\footnotetext{
${ }^{2}$ Sky maps for 100 randomly placed observers in this new simulation can be found here: https://drive.google.com/drive/folders/ 1LIPmL05ENjLq4EnDBtLEyMAY2pRrcKm9?usp=sharing.
} 


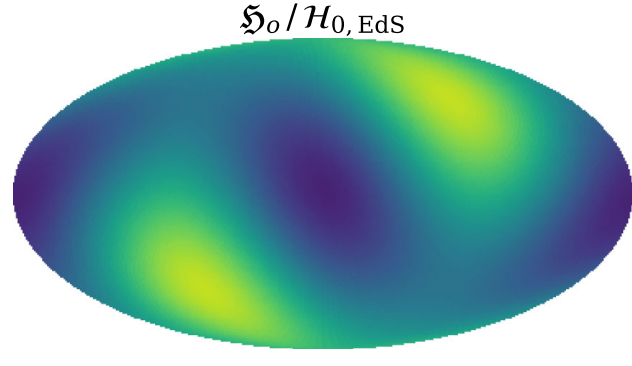

0.98

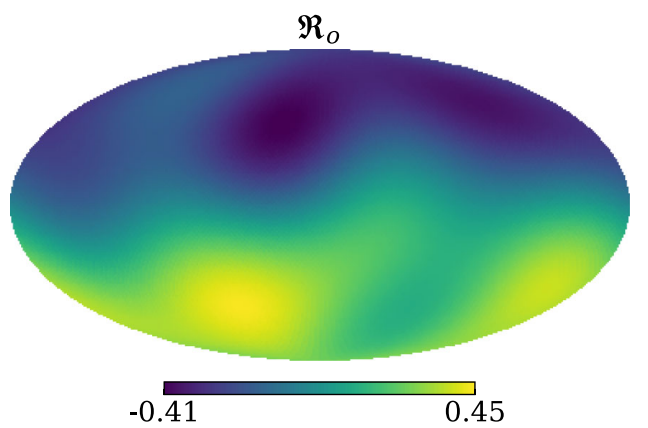

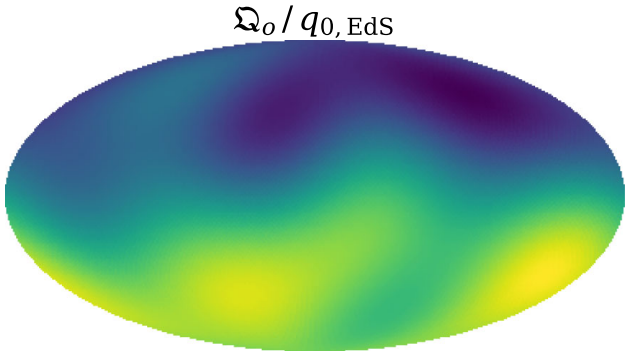

0.15

1.85

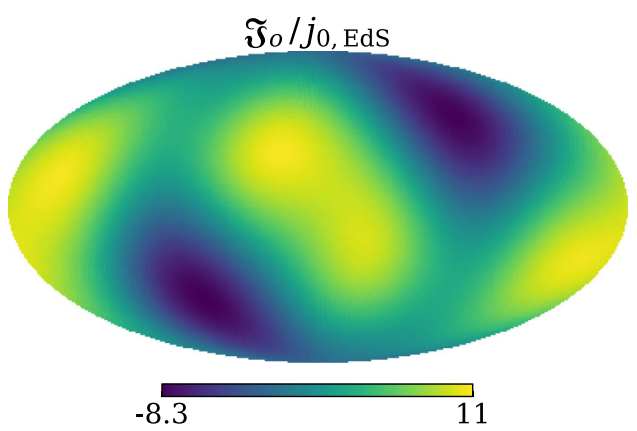

FIG. 2. Sky maps of the observational effective Hubble, deceleration, curvature, and jerk parameters (top-left to bottom-right panels, respectively) for an example observer in our updated simulation with $200 \mathrm{~h}^{-1} \mathrm{Mpc}$ coarse-graining scale.
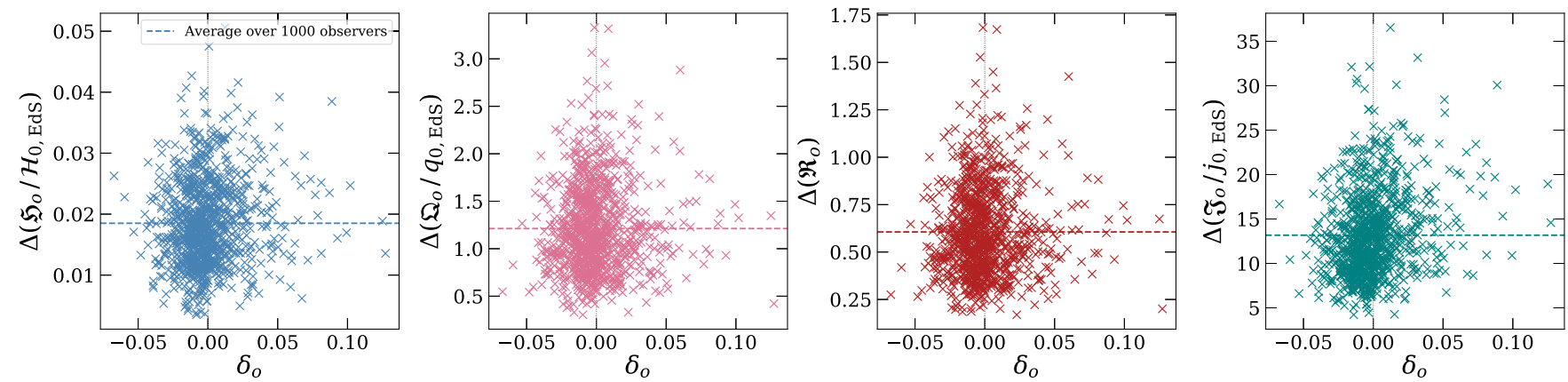

FIG. 3. Panels (left to right) show the maximal sky-variance for the effective Hubble, deceleration, curvature, and jerk parameters relative to their Einstein-de Sitter (EdS) counterparts in the updated simulation with $200 \mathrm{~h}^{-1} \mathrm{Mpc}$ coarse-graining scale. This is to be compared with Fig. 4 in $\mathrm{MH} 21$.

TABLE I. Anisotropy of effective cosmological parameters across typical and extreme observers' skies in our updated simulations. We show the mean and maximum sky deviation, $\Delta$, over 1000 observers in simulations with effective smoothing lengths of 100 and $200 h^{-1}$ Mpc. This is for comparison with Table I in MH21.

\begin{tabular}{|c|c|c|c|c|}
\hline & \multicolumn{2}{|c|}{$100 h^{-1}$ Mpc smoothing } & \multicolumn{2}{|c|}{$200 h^{-1}$ Mpc smoothing } \\
\hline & Observer mean & Observer max & Observer mean & Observer max \\
\hline$\Delta\left(\mathfrak{S}_{o} / \mathcal{H}_{0, \text { EdS }}\right)$ & 0.068 & 0.23 & 0.019 & 0.051 \\
\hline$\Delta\left(\mathfrak{Q}_{o} / q_{0, \mathrm{EdS}}\right)$ & 8.5 & 27 & 1.2 & 3.3 \\
\hline$\Delta\left(\mathfrak{R}_{o}\right)$ & 4.3 & 13 & 0.61 & 1.7 \\
\hline$\Delta\left(\mathfrak{\Im}_{o} / j_{0, \text { EdS }}\right)$ & 193 & 675 & 13 & 37 \\
\hline
\end{tabular}



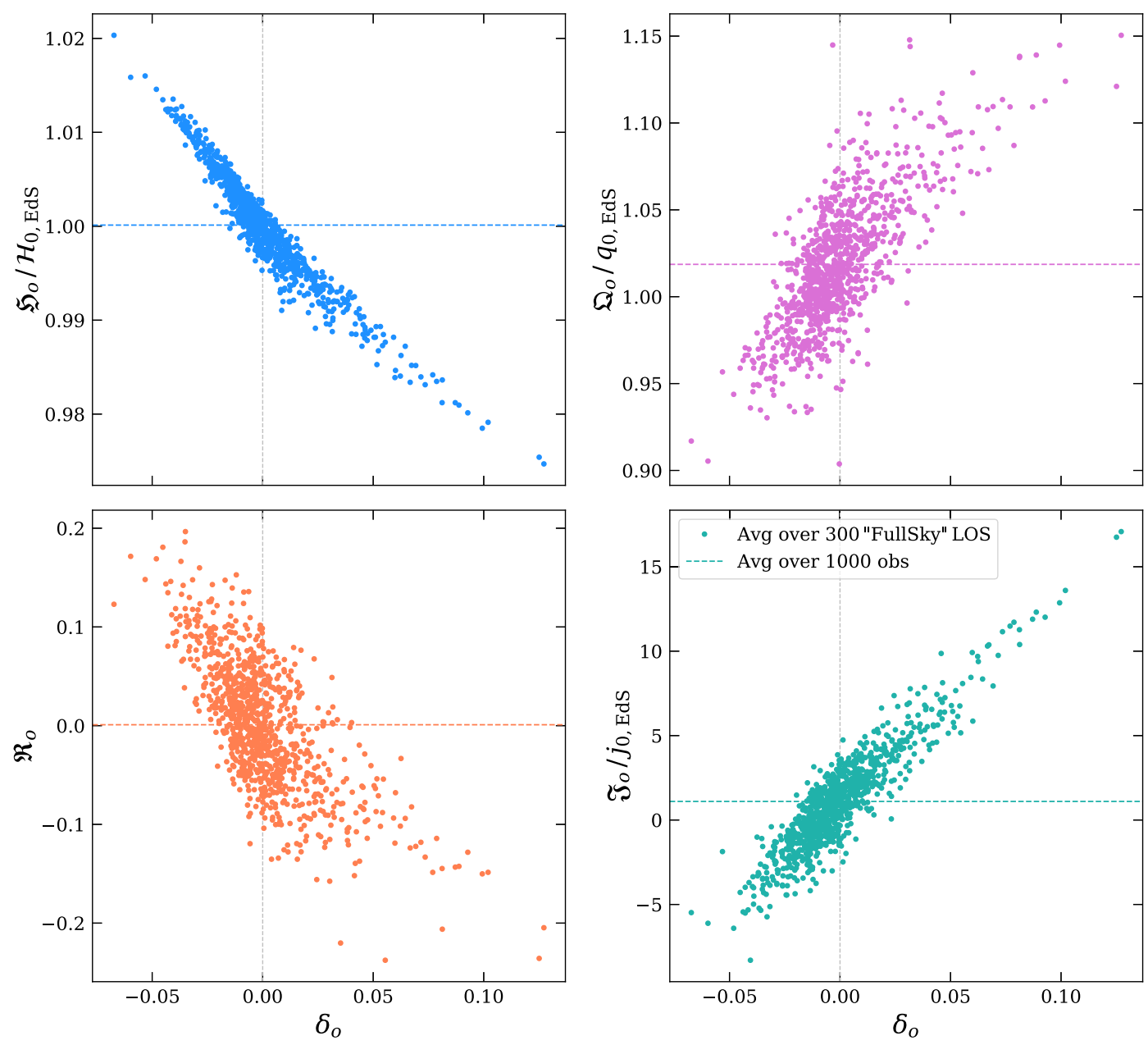

FIG. 4. Effective cosmological parameters relative to their EdS counterparts (panels) calculated in our updated simulation with $200 h^{-1} \mathrm{Mpc}$ coarse-graining scale. Points show individual observers, with local density $\delta_{o}$, averaged over 300 randomly chosen lines of sight across their whole sky. This is for comparison with Fig. 5 in MH21.

Typical observers in our new simulations measure $120 \%$ (850\%) variance in the deceleration parameter across their sky, and $61 \%(430 \%)$ variance in the curvature parameter for coarse-graining scales of $200(100) h^{-1}$ Mpc. Typical maximal sky variance in the effective Hubble parameter is $\sim 2 \%$ (7\%) for the same scales, although extreme observers measure up to $5 \%(23 \%)$ sky variance.

\section{Sky averages of observational effective parameters}

Here we reassess the sky averages for individual observers in our updated simulations. We calculate the average over "FullSky" and "HalfSky" distributions of 300 lines of sight for 1000 randomly placed observers.

Figure 4 shows the sky average for 1000 observers (points) of the observational effective parameters (panels), averaged over 300 "FullSky" lines of sight. Dashed lines show the average over all observers. This is to be compared with Fig. 5 in $\mathrm{MH} 21$. Our results here are consistent with $\mathrm{MH} 21$ for the same values of $\delta_{o}$, with the exception of the curvature parameter which we comment on below.

Figure 5 shows the sky average for 1000 observers (points) with 300 lines of sight from the "HalfSky" sampling (randomly drawn directions from one half of each observers' sky). This is for comparison with Fig. 6 in MH21. The change in cosmic variance moving between the "FullSky" and "HalfSky" samplings here is less drastic than MH21, which can be attributed to lower amplitudes of anisotropy for our new observers.

One notable difference here is that we now see an anticorrelation between the effective curvature parameter and the local density contrast, compared to a positive correlation seen in MH21. This anticorrelation is expected when the effective curvature parameter is dominated by the contribution from the 3-curvature scalar of the space orthogonal to the observer 

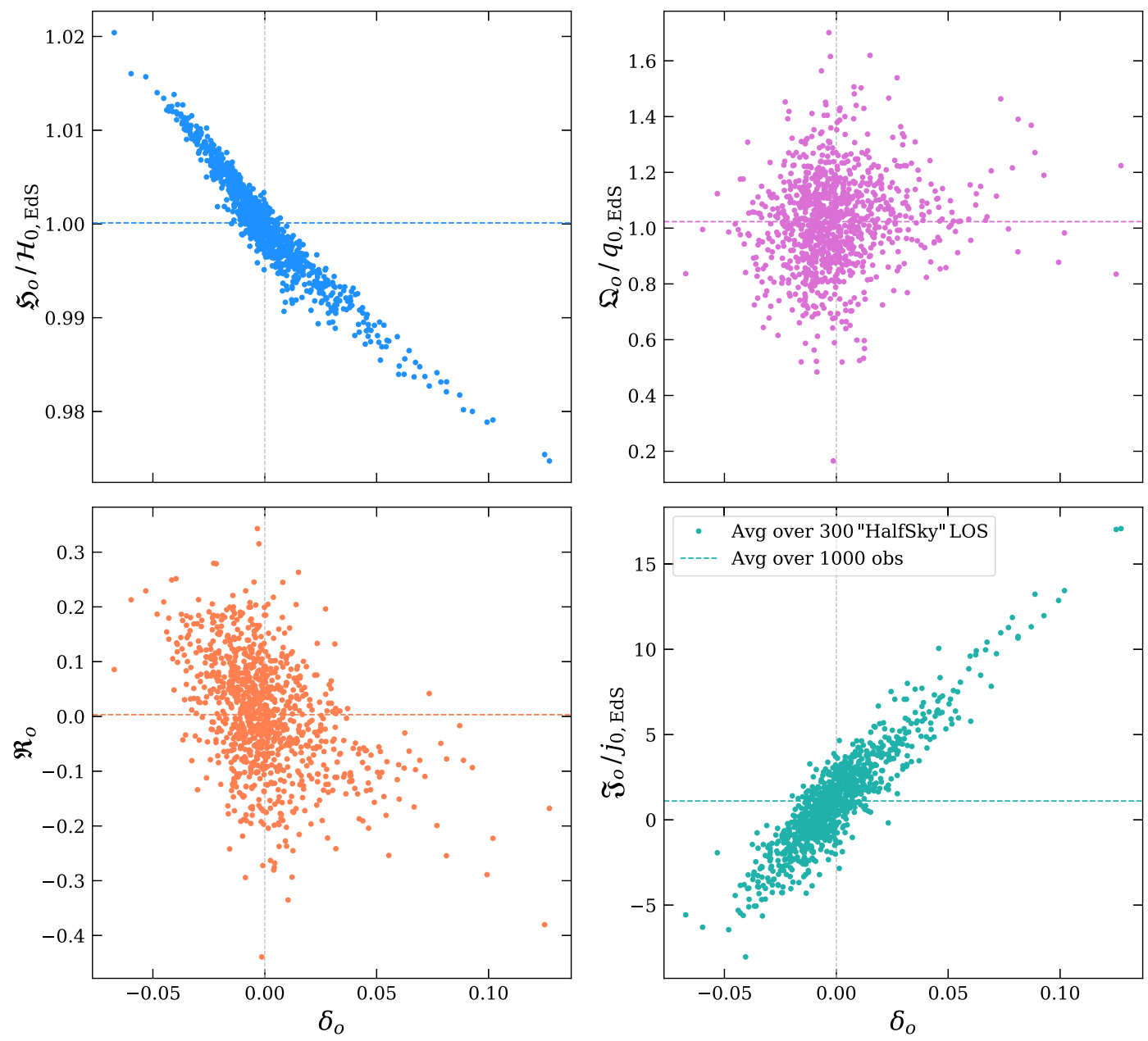

FIG. 5. Effective cosmological parameters relative to their EdS counterparts (panels) calculated in our updated simulation with $200 h^{-1} \mathrm{Mpc}$ coarse-graining scale. Points show individual observers, with local density $\delta_{o}$, averaged over 300 randomly chosen lines of sight across half of their sky. This is for comparison with Fig. 6 in MH21.

TABLE II. Cosmic variance of observational effective cosmological parameters in our updated simulations. We show standard deviations of the distribution of sky averages - for the "FullSky" and "HalfSky" distributions_for 1000 observers placed in updated simulations with effective smoothing lengths of 100 and $200 \mathrm{~h}^{-1} \mathrm{Mpc}$.

\begin{tabular}{lcccc}
\hline \hline & \multicolumn{2}{c}{$100 h^{-1}$ Mpc smoothing } & \multicolumn{2}{c}{$200 h^{-1}$ Mpc smoothing } \\
\cline { 2 - 3 }$\sigma($ sky avgs $)$ & "FullSky" & "HalfSky" & & "FullSky" \\
\hline$\sigma\left(\mathfrak{H}_{o} / \mathcal{H}_{0, \text { EdS }}\right)$ & 0.022 & 0.021 & 0.0057 & 0.0057 \\
$\sigma\left(\mathfrak{Q}_{o} / q_{0, \text { EdS }}\right)$ & 0.19 & 1.2 & 0.038 & 0.17 \\
$\sigma\left(\mathfrak{R}_{o}\right)$ & 0.22 & 0.64 & 0.064 & 0.11 \\
$\sigma\left(\mathfrak{\Im}_{o} / j_{0, \text { EdS }}\right)$ & 43 & 43 & 2.9 & 2.9 \\
\hline \hline
\end{tabular}

congruence. This is the case when gradients of the 4-acceleration, quadratic shear, and vorticity ${ }^{3}$ are subdominant relative to the 3-curvature scalar.

We summarise the standard deviation across all 1000 observers (cosmic variance) of all effective parameters in Table II. We present both "FullSky" and "HalfSky" samplings and for both simulations with 100 and $200 h^{-1}$ Mpc coarse-graining scales. This is to be compared with Table II in MH21.

\footnotetext{
${ }^{3}$ In case of congruences with vorticity, we note that a 3-curvature scalar, generalizing the 3-Ricci scalar of an irrotational flow, can be defined in the tangent spaces orthogonal to the congruence flow lines [3].
} 


\section{CONCLUSIONS}

There was a bug in the code which produced the initial data for the simulations used in the analysis of low-redshift anisotropies in MH21. This had a significant effect on the resulting density field at $z \approx 0$. In this erratum we fixed the bug and presented all main results from $\mathrm{MH} 21$ for the corrected simulations.

We summarize our main conclusions as follows:

(i) Qualitatively, our results are unchanged. We find the same multipoles dominating the anisotropic signatures for the effective parameters as in MH21.

(ii) Our corrected simulations have smaller typical density contrasts by a factor of $\sim 3$, resulting in an overall reduction in the amplitude of anisotropy measured by the observers.

(iii) Due to the factor of $\sim 3$ reduction in density contrasts, our results for $100 \mathrm{~h}^{-1} \mathrm{Mpc}$ smoothing lengths now more closely reflect our results for $200 h^{-1}$ Mpc smoothing lengths presented in MH21 (see the Appendix).

Our conclusion that an unfairly sampled sky may bias observers' measurements of cosmological parameters still stands, and this remains an important issue to be investigated in more detail in low-redshift data.

The authors would especially like to thank Julian Adamek for initially noticing this error, and H. J. M. would like to further thank him for his patience and time spent helping us to ensure our new simulations were correct. We would also like to thank Ruth Durrer, Martin Kunz, and Chris Clarkson for helpful discussions during the process of correcting our simulations. H. J. M. appreciates support received from the Herchel Smith Postdoctoral Fellowship Fund. This work is part of a project that has received funding from the European Research Council (ERC) under the European Union's Horizon 2020 research and innovation programme (grant agreement ERC advanced Grant No. 740021-ARTHUS, PI: Thomas Buchert). This work used the DiRAC@Durham facility managed by the Institute for Computational Cosmology on behalf of the STFC DiRAC HPC Facility [4]. The equipment was funded by BEIS capital funding via STFC capital Grants No. ST/ P002293/1, No. ST/R002371/1, and No. ST/S002502/1, Durham University and STFC operations Grant No. ST/R000832/ 1. DiRAC is part of the National e-Infrastructure.

\section{APPENDIX: PLOTS FOR $100 \boldsymbol{h}^{-1}$ MPC SMOOTHING}

In this appendix we present the maximal sky variance and sky averages over both "FullSky" and "HalfSky" distributions for the updated simulation with $100 \mathrm{~h}^{-1} \mathrm{Mpc}$ smoothing scale. As stated in our conclusions, because of the reduction in typical density contrasts in the updated simulations, the results we present here for $100 \mathrm{~h}^{-1} \mathrm{Mpc}$ smoothing scales are closer to the results presented in MH21 for a $200 \mathrm{~h}^{-1}$ Mpc smoothing scale.

Figure 6 shows the maximal sky variance $\Delta$ for each effective cosmological parameter relative to its EdS counterpart (panels), as a function of the local density at the observer's position. Dashed lines show the average over all 1000 observers.

Figure 7 shows the sky average for 1000 observers (points) over a "FullSky" distribution of 300 lines of sight for each effective cosmological parameter relative to its EdS counterpart (panels). We show sky averages as a function of the observer's local density, $\delta_{o}$. Dashed lines again show the average over all 1000 observers.

Figure 8 shows the sky average of the effective parameters for a "HalfSky" distribution of 300 lines of sight for 1000 observers. Dashes lines are the average over all observers, and black dot-dashed lines show the $\Lambda$ CDM value of some parameters.
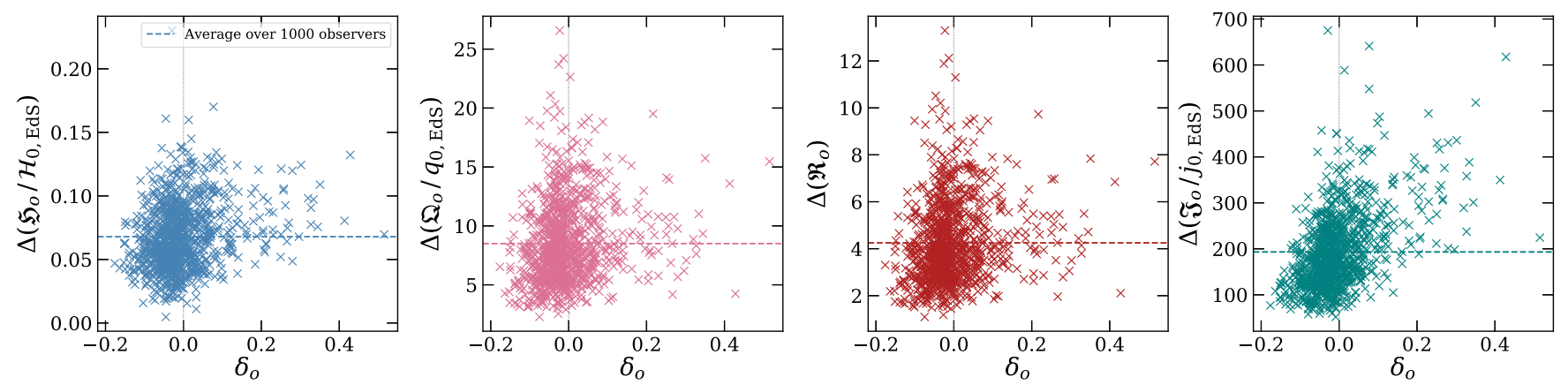

FIG. 6. Panels (left to right) show the maximal sky variance for the effective Hubble, deceleration, curvature, and jerk parameters relative to their EdS counterparts in the updated simulation with $100 \mathrm{~h}^{-1} \mathrm{Mpc}$ coarse-graining scale. 

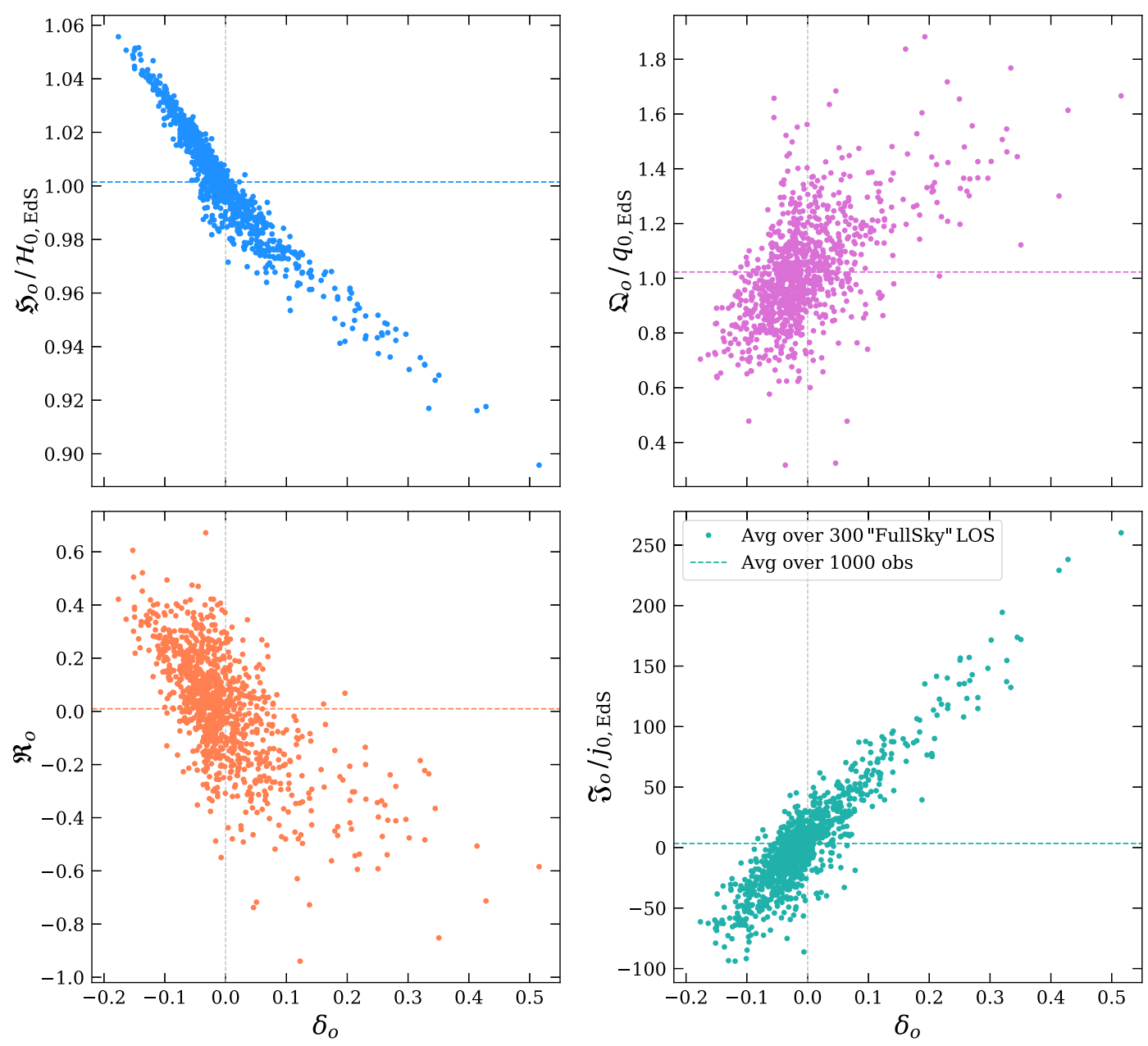

FIG. 7. Effective cosmological parameters relative to their EdS counterparts (panels) calculated in our updated simulation with $100 h^{-1}$ Mpc coarse-graining scale. Points show individual observers, with local density $\delta_{o}$, averaged over 300 randomly chosen lines of sight across their whole sky ("FullSky" distribution). 

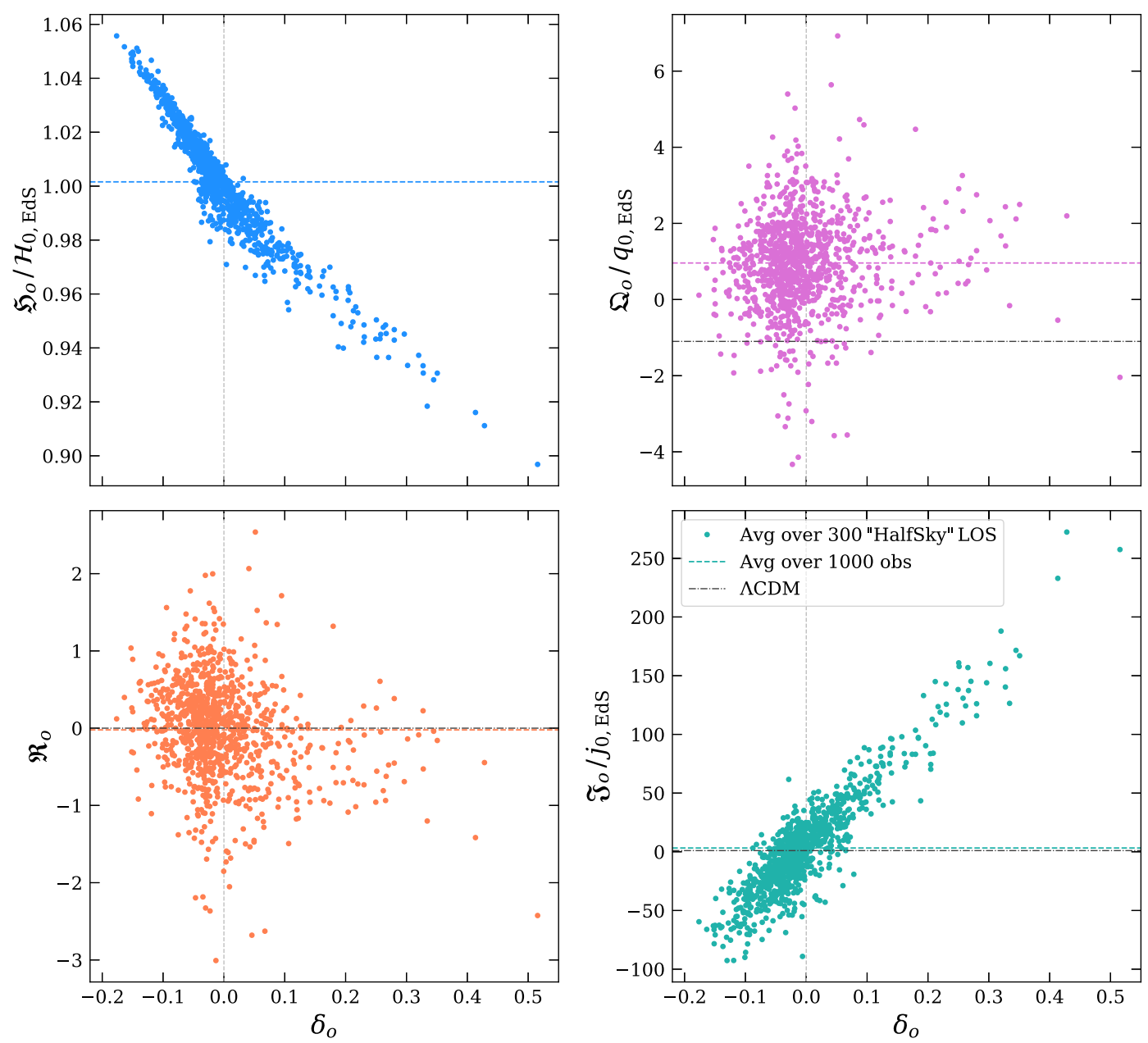

FIG. 8. Effective cosmological parameters relative to their EdS counterparts (panels) calculated in our updated simulation with $100 h^{-1} \mathrm{Mpc}$ coarse-graining scale. Points show individual observers, with local density $\delta_{o}$, averaged over 300 randomly chosen lines of sight across half of their sky ("HalfSky" distribution).

[1] H. J. Macpherson and A. Heinesen, Luminosity distance and anisotropic sky-sampling at low redshifts: A numerical relativity study, Phys. Rev. D 104, 023525 (2021).

[2] H. J. Macpherson, D. J. Price, and P. D. Lasky, Einstein's Universe: Cosmological structure formation in numerical relativity, Phys. Rev. D 99, 063522 (2019).

[3] G. F. R. Ellis, M. Bruni, and J. Hwang, Density-gradient-vorticity relation in perfect-fluid Robertson-Walker perturbations, Phys. Rev. D 42, 1035 (1990).

[4] www.dirac.ac.uk. 